\title{
Announcement
}

\section{Free Color Online in Cambridge University Press Journals}

Cambridge University Press is pleased to announce a new benefit to authors of papers in selected journals. Beginning in 2008, all figures submitted to CUP journals will be published in color on Cambridge Journals Online free of charge. Our color policy is based on a model previously instituted by The Electrochemical Society.

\section{Author Requirements}

It is not necessary for authors to indicate that a figure should be displayed in color online. CUP will assume that any author who submits figures in color wants and agrees to their being produced in color online. It is the author's responsibility to declare otherwise. Color figures must be submitted before the paper is accepted for publication, and cannot be received later in the process. Authors cannot submit two versions of the same figure, one for color and one for black and white; only one version can be submitted.

Authors need to carefully consider the following when submitting figures in color that will be published in color online only:

The colors chosen must reproduce effectively and the colors should be distinguishable when printed in black and white.

The descriptions of figures in text and captions must be sufficiently clear for both online and print copy.

When submitting figures to be in color online only, authors must include the phrase «color online» in the figure captions. This is the author's responsibility. Here is an example of a figure caption for a color online only figure:

Figure 1. (Color online) Experimental (red dotted curve) and simulated (blue solid curve) X-ray diffraction spectra.

\section{Exceptions to Free Color Online}

Color figures submitted to a CUP journal will be published in color online free of charge with the following exceptions:

The color figure file is deemed unusable due to production standards or poor color quality and must be converted to black and white.

The author gives explicit instructions to convert the color figure to black and white.

The author gives explicit instructions that the figure should be published in color both in print and online, and agrees to pay the additional charge associated with color printing. This must be conveyed to the journal's Production Office at CUP no later than the proof correction stage.

\section{What to Expect}

Authors will see these color figures when viewing their author page proofs on screen. Authors should always print their page proofs in black and white to see how they will appear in print. Authors will NOT be allowed to submit color figures to replace black and white figures in the page proof stage.

\section{How to Ensure Color Online}

To maximize the probability that figures will be published in color online and also print as good quality black and white or grayscale graphics, authors are encouraged to follow these figure submission guidelines:

Submit a color graphic in Tagged Image File Format (.tiff).

Submit color graphics with a resolution of at least 300 dpi (600 dpi if there is text or line art in the figure).

Submit figures sized to fit the actual column or page width of the journal so that reduction or enlargement is not necessary.

Submit multipart figures in one single electronic file.

Consult this website for further information about file formats and resolution:

http://dx.sheridan.com/ 